\title{
Presymptomatic Testing
}

National Cancer Institute ( $\mathrm{NCl})$

\section{Source}

National Cancer Institute (NCI). Presymptomatic testing.

Genetic analysis of an asymptomatic or unaffected individual who is at risk of a specific genetic disorder. 